\title{
Present situation of research and Application of High Intensity Focusing Ultrasonic Therapy
}

\author{
Liu $\mathrm{Li}^{1, a^{*}}$, Zhang Song ${ }^{2, b}$
}

The faculty of Mechanical Engineering and Mchanics, Ningbo University, Ningbo city of Zhejiang

Province, P. R. China; 315211

aliuli@nbu.edu.cn, b373900849@qq.com

Keyword: High intensity focusing ultrasonic, Transducer, Non-invasive temperature measurement Abstract. High intensity focused ultrasound (HIFU) is the fourth brand-new and efficient means to cure tumour acknowledged by the medical field. China is regarded as one of countries applying HIFU to clinical oncotherapy earliest in the world. However, a considerable part of HIFU equipments which have been put on the market leave unused because accidental injuries such as ambustion to the body surface, tumour tissue residues in the target section or damages to normal tissues are often caused in HIFU clinical treatment. In the paper, ultrasonic focusing method, temperature measurement technique of high-intensity focusing ultrasonic therapy is introduced; studies of current situations of cell and multiplex array focusing transducers and their existing problems are focally analyzed and illustrated based on analyzing challenges faced by HIFU treatment at present. This study suggested that phased array was theoretically easy for realizing accurate control of computer, however, unbeneficial factors and engineering technical problems still exist.

\section{Introduction}

HIFU focuses extracorporeal low-energy ultrasound in small focal area (or focal spot) so that nidus tissues such as tumour and hyperplasia in the focal area can accept irradiation within short time (usually 1 10s) and heat up to over $65^{\circ} \mathrm{C}$ rapidly and will not generate irreversible coagulative necrosis [1][2] by virtue of visuality, tissue penetrability and focusing of ultrasound and through certain form of ultrasonic focusing transducer. High intensity focusing ultrasonic(HIFU)technique, a local treatment means with minimal invasion or without invasion, has been acknowledged by the medical circle as the 4th brand-now and high-efficient means for curing tumours after operation, chemotherapy and radiotherapy and has become a hotspot and a focus highly attended by domestic and foreign medical circles.

The dissertation working group led by Professor Shou Wende from Shanghai Jiaotong University has conducted systematic theoretic researches, technical breakthroughs and experimental verifications about HIFU sound field and measurement of acoustical power, making innovations as follows: educing the relation between the acoustical power and the redial force of several typical focusing transducers used in HIFU treatment; successfully studying and developing measurement of heat-resistant bondacust target of HIFU acoustical power by radiation method.

\section{Application Status}

China is one of earliest countries applying HIFU to clinical oncotherapy and it has been 13 years till now. Till 2009, there have been about 10 enterprises in the world which officially produce and sell HIFU treatment equipments and 7 were from China [3]: Chongqing Haifu Company, Beijing Deyuan Company, Shanghai Aishen Company, Mianyang Sonic Company, Xidi Compnay of Shanghai Jiaotong University and so on. However, it is said in several channels that a great deal of sold and released HIFU equipments have been left unused because invalidity, complication and accidents are not rare now and complications such as skin burn, neurotrosis and residue of tumor tissues in the target section. It has been found by experts that we have to reduce the preset power even interrupt the treatment due to serious pain outside the focal area in HIFU treatment. HIFU still belongs to medical technique and apparatus under mature development now and must face severe 
challenges for ensuring curative effect and avoiding serious temperature damage complication [3].

\section{Ultrasonic Focusing Method}

HIFU technique in earlier times is to acquire focusing ultrasonic wave by concave quartz crystal or plane quartz crystal and polystyrene sound lens. In recent years, quartz crystal has been replaced by piezoelectric ceramics chip with high electroacoustic efficiency [2], its focusing wary has also trended to diversity according to actual demands. Focusing methods stated above can divide HIFU transducers into two types: cell array focusing transducer and multiplex array focusing transducer. Both have a round hole in the centre for placing the B ultrasonic monitoring probe so as to find accurate position of the nidus and the monitoring process.

\section{Current Situation about Studying Transducer}

Two types of cell array focusing transducer are usually used: plane vibrator sound lens and concave spherical shell self-focusing (the simple graph of the focusing field is shown in figure 1 and 2).. Concave spherical shell self-focusing has better properties than plane vibrator sound lens and is more suitable for applying HIFU to cure tumour [4]. In order to promote reliability and safety of treatment, it is quite necessary to predict sound field formed by HIFU transducer. Traditional HIFU applies cell array focusing with fixed focal point; while it is inconvenient to apply mechanical method; it is slow and restricts its application [5].

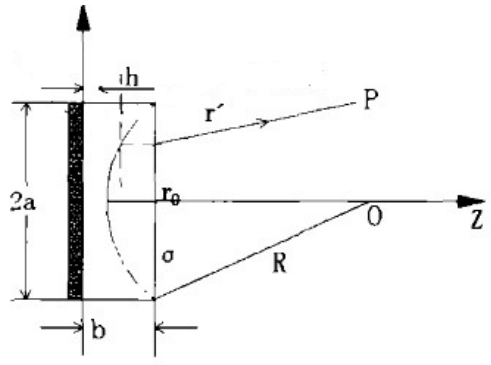

Figure 1 the sound lens focusing field

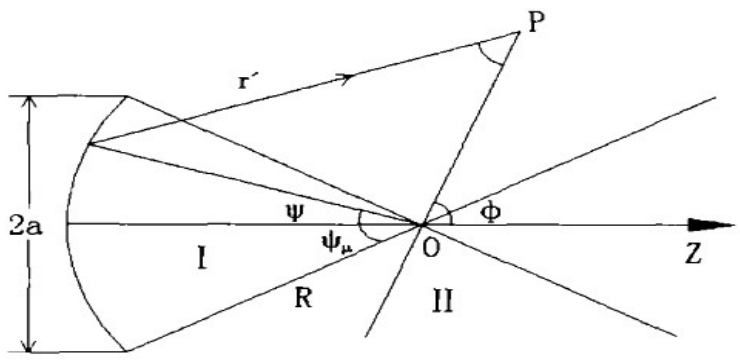

Figure 2 the concave spherical self-focusing field Focusing way of multiplex array transducer can be divided into [4]: multiplex coherent focusing transducer, multiplex non-coherent focusing transducer and electronic scanning transducer or phased focusing transducer, among which phased focusing realizes variable focal length and multiple focal points through accurately controlling amplitudes and phases of the ultrasound wave launched from different array elements (multiple probes) on the array transducer by computer. In recent years, It has been a hot research topic and researchers have designed phased arrays with different shapes.

HIFU phased array transducer settlers many problems in treatment; for example, volume of the focal spot generated by the cell array focusing transducer is very small and it will take a long time to cure big tumour. In order to realize intensity difference inside and outside the focal spot, some probes are added in some products to participate in the focusing (over 500 probes are added in some products). Then, consistence in properties of single probes are highly requested; quality of the focal spot is hardly controlled or there are several focal spots; medical accidents caused by injury or other tissues often occur in earlier clinical application of the product [6]. Therefore, phased array is theoretically easy for realizing accurate control of computer; however, unbeneficial factors and engineering technical problems such as complex circuit, large influences of nonlinear effect and it is hard to generate focusing acoustic beams with sufficient intensity in deep tissues still exist; meanwhile, high requests about processing accuracy and installation technology of transducer are proposed.

\section{Temperature Measurement Method in HIFU}

In order to kill nidus cell without damage to normal tissues, it is very important to measure and 
control temperature of tissues in HIFU treatment. Researchers such as Xiong Liulin from the People's Hospital of Peking University has studied ideal temperature and action time of high-intensity focusing ultrasonic focal area, which ensures curative effect of domestic heat melting treatment and offers experimental basis to reduce damages to the upper sound tissue and tissues surrounding the focal spot as much as possible.

HIFU temperature measurement technique can be divided into invasive temperature measurement and non-invasive temperature measurement; at the earlier state, invasive temperature measurement was applied mostly in clinic, namely invasion measurement by a temperature measuring needle. Invasive measurement refers to inserting temperature sensors such as thermocouple and thermistor into the measured part for direct measurement; then, temperature of the measured point is acquired and it is hard to measure temperature distribution [7], which causes pains to patients and holds the danger to transfer cancer cells. There have been lots of researches and reports about invasive temperature measurement and distribution of its temperature field [8] both at home and abroad. Non-invasive temperature measurement has been focused in recent years; many methods have been proposed in researches such as microwave radiation, CT, ultrasound and MRI (Magnetic Resonance Imaging). Application of CT is limited because it has radiation and human body is not suitable for receiving it for a long time; ultrasound and MRI are two imaging devices usually used at home and abroad for guiding and monitoring HIFU treatment and are also hot research topics.

Ultrasonic Non-invasive Temperature Measurement. Ultrasonic non-invasive temperature measurement refers to launching ultrasound wave to hypodermis, receiving echo from these tissues, acquiring ultrasonogram by different mathematical algorithms, describing temperature field of tissues and further inferring temperature change of the measured part [9]. Ultrasonic imaging has mature technique and is economical and easy for combining HIFU devices; thus, it is an effective means and method for monitoring orientation and curative effect of HIFU treatment by $B$ ultrasonic.

However, ultrasonic imaging, as a means for supervising and guiding HIFU treatment, has many defects, which has been analyzed and illustrated by famous experts such as G. terHaar and proved in ordinary clinical cases. Major reasons take on as follows: the average value of the gray level of B ultrasonic images is just in positive relevance with the radiation dosage of HIFU in certain scope, which turns bigger along with larger coagulative necrosis areas of tissues; while there is an obvious boundary between the average values of the gray level of the damage area of different tissues. When the dosage is high enough, the average value will not turn bigger along with increase in dosage. When the ultrasonic focal point is near the threshold temperature $\left(56^{\circ} \mathrm{C}\right.$, the threshold temperature or the toxicology temperature of albuminous degeneration), coagulative necrosis of the tissue is not reflected as enhancement of the gray scale; even though the target is lost even runs out of the nidus, it cannot be found in B ultrasonic; obvious enhancement of the gray scale can just be found when the temperature is so high that water in tissues is boiling with heat cavitation phenomenon; in that case, although melting is reached, the wrong burning position is irreversible and side effect of high temperature cannot be neglected [5]. Treatment machines researched and developed at home can just compare changes in the gray level of images from B ultrasonic and judge whether coagulative necrosis occurs and its position is correct [10].

MRI Non-invasive Temperature Measurement. MRI with high resolution as a strong point can clearly shows the anatomy image of the nidus and surrounding areas before treatment; exerting exploratory sound intensity, and temperature of tissues near the focal point exceeds physiological temperature; MRI can show and detect it; thus, we can correct the deviating position, calculate and adjust the treatment dosage according to detection result at any time; furthermore, we can judge the melting effect and conduct monitoring and guide by virtue of changes in images after the temperature exceeds the threshold value appropriately and maintains a certain period of time [5]. This technique is mainly grasped by America, Germany and Japan and is applied to cure myoma of uterus at present. In October, 2004, American FDA approved that the focusing ultrasonic treatment system navigated by MRI is a kind of new technique for treating myoma of uterus without invasion 
completely; its effectiveness and safety have been proved in lots of clinic [11]. Technical breakthroughs in this aspect are mainly undertaken by enterprises so as to realize MRI temperature measurement technique in domestic HIFU devices; for example, Chongqing Haifu Co., Ltd. and Germany Siemens Company cooperated to research and develop HIFU treatment system navigated by MRI(magnetic resonance guided high intensity focused ultrasoun, MRgHIFU) [12][13].

MRI temperature measurement is limited because it is rather sensitive to motions caused by breath, heartbeat and body movement etc so as to cause errors in temperature measurement [9]. Besides, MRI temperature measurement restricts curing most tumours in the midsection and behind the peritoneum due to influences of breath; in addition, it is expensive and needs high requests in compatibility for connecting with MRI system [11]; thus, it has not been extensively applied in clinic.

\section{Summary}

Since HIFU was established, progress has been made in scientific researches and technical breakthroughs step by step, which has been further strengthened since 1990s. Efforts have been made to study and break through medical ultrasonic physics, biological and physical basis, engineering technique and clinic in lots of western countries. In China, HIFU still belongs to medical technique and apparatus under mature development now and must face severe challenges for ensuring curative effect and avoiding serious temperature damage complication [3].

\section{Acknowledgements}

This work is supported by Scientific Research Fund of Zhejiang Provincial Education Department (Y201224508/2012), Scientific Research Fund of Zhejiang Provincial Education Department (Y201224508/2012).

\section{References}

[1] H.B. Su, J.Z. Zou, and Z.B. Wang: Chin J Hepatobiliary Surg, Vol. 17 (2011) No.3, pp. 271-272. (In Chinese)

[2] Y.H. Ji, Q.L. Li, Y. Yang, et a1.: Chinese Journal of Medical Physics, Vol. 25 (2008) No.4, pp. 759-762,780. (In Chinese)

[3] F.Q. Niu, Y. Cheng and C.G. Zhu: China Medical Device Information, Vol. 15 (2009) No.5, pp.32-39. (In Chinese)

[4] L. Zhai; F.C. Sun.; X.P. Qian, et a1.: Medical Equipment Journal, 2007(01), pp.8-10. (In Chinese)

[5] C. Liu, W. Wei, Y.L. Jiang, et a1.: The 8 (TH) China Nondestructive Testing Congress (SuZhou, September23-24, 2003). pp.203-208.

[6] J.Q. Zhang: China Medical Device Information, Vol. 14 (2008) No.4, pp.17-18,35. (In Chinese)

[7] S.C. Wu, Y.P. Bai, Q. Nan, et a1.: Foreign Medical Sciences Biomedical Engineering, Vol. 25 (2002) No.1, pp.5-38. (In Chinese)

[8] F.Q. Li, Z.B. Wang, Y.H. Du, et a1.: Chinese Journal of Biomedical Engineering, Vol. 22 (2003) No.4, pp.321-325. (In Chinese)

[9] L.X. Jiang and B. Hu: Technical Acoustics, Vol. 25 (2006) No.1, pp.43-47. (In Chinese)

[10] L.Z. Fan, F. Luo, D.Y. Yu, et a1.: Chinese Journal of Medical Instrumentation, Vol. 29 (2005) No.2, pp.115-119,111. (In Chinese)

[11] M. He, L. Zhang and J. Bai: Ultrasound in Clin Med, Vol. 12 (2010) No.1, pp.39-42. (In Chinese)

[12] Y.H. Xu, Z.X. Fu, L.X. Yang, et al.: Journal of Interventional Radiology, Vol. 19 (2010) No.11, pp.869-874. (In Chinese)

[13] G.J. Teng: Journal of Interventional Radiology, Vol. 19 (2010) No.11, pp.841-842. (In Chinese) 